\title{
Kepler's Laws, Newton's Laws, and the Search for New Planets
}

\section{Robert Osserman}

Introduction. One of the high points of elementary calculus is the derivation of Kepler's empirically deduced laws of planetary motion from Newton's Law of Gravity and his second law of motion. However, the standard treatment of the subject in calculus books is flawed for at least three reasons that I think are important.

First, Newton's Laws are used to derive a differential equation for the displacement vector from the Sun to a planet; say the Earth. Then it is shown that the displacement vector lies in a plane, and if the base point is translated to the origin, the endpoint traces out an ellipse. This is said to confirm Kepler's first law, that the planets orbit the sun in an elliptical path, with the sun at one focus. However, the alert student may notice that the identical argument for the displacement vector in the opposite direction would show that the Sun orbits the Earth in an ellipse, which, it turns out, is very close to a circle with the Earth at the center. That would seem to provide aid and comfort to the Church's rejection of Galileo's claim that his heliocentric view had more validity than their geocentric one.

Second, by placing the sun at the origin, the impression is given that either the sun is fixed, or else, that one may choose coordinates attached to a moving body, inertial or not. However, Newton's equations in their usual form hold precisely in inertial coordinates. Furthermore, it is an immediate consequence of Newton's Laws that the center of gravity of the two bodies can serve as the origin of an inertial coordinate system. One then finds that both the Earth and the Sun describe elliptical paths with a focus at the center of gravity of the pair.

Third, by considering only the displacement vector, the treatment misses an opportunity to present one of the greatest recent triumphs of Newton's derivation of Kepler's Laws: the discovery of extrasolar planets during the past decade, none of which has been observed directly, but whose existence and orbital parameters have been deduced by detecting and analyzing the motion of the stars that they orbit.

Two other considerations make the correct way of treating the subject particularly desirable. First, the current treatment for the displacement vector could be left as is, and only a little algebra is needed to show that the same vector referred to the center of gravity is a constant scalar multiple of the one referred to the sun. Second, it connects with an exciting area of current research in astronomy where the methods of elementary calculus have direct application.

The purpose of this note is to spell out the slight modifications to the standard treatment of Kepler's Laws that are needed to obtain the additional and accurate information, and to provide a user's guide to the website http://exoplanets.org where a list of all the extrasolar planets discovered so far is maintained and constantly updated.

The Basic Equations. Let $\mathbf{r}(t)$ be a (3-dimensional) vector function of the parameter $t$, and suppose that $\mathbf{r}(t)$ satisfies the differential equation

$$
\mathbf{r}^{\prime \prime}(t)=-c \mathbf{r}(t) / r^{3}
$$


where $c$ is a positive constant and $r$ is the magnitude of the vector $\mathbf{r}$. Then the following statements hold, and are generally proved in calculus books under the general rubric of "Newton's laws imply Kepler's Laws."

$\mathrm{P} 1$. If $\mathbf{r}$ is viewed as a position vector whose base point is at the origin, then its endpoint lies on a plane curve that is either along a ray through the origin, or else a conic section with focus at the origin. In the latter case, if the solution curve is bounded, it must be an ellipse. (Kepler's First Law.)

P2. The vector $\mathbf{r}(t)$ sweeps out equal areas in equal times; that is, in equal $t$ intervals. (Kepler's second law.)

P3. If $T$ is the period, the $t$-interval in which $\mathbf{r}(t)$ traverses the ellipse once, and if $a$ is the semi-major axis of the ellipse, then $T$ and $a$ are related by the equation

$$
T^{2} / a^{3}=d,
$$

where

$$
d=4 \pi^{2} / c .
$$

In the two-body problem, one asks for the motion determined by the gravitational force between two bodies, assuming one can neglect all other forces, including those of other more distant bodies whose effect on these two would be secondary. Let us denote the two bodies as the earth and the sun, and let $\mathbf{r}$ be the displacement vector from the sun to the earth. Then, the vector function $\mathbf{r}(t)$, where $t$ is time, does indeed satisfy equation (1) for a suitable value of the constant $c$, but not the equation that one finds in calculus books. Namely, if $m$ is the mass of the earth, and $M$ is the mass of the sun, then the gravitational force exerted by the sun on the earth is $-G M m \mathbf{r} / r^{3}$, where $G$ is the gravitational constant. The claim is then made that by Newton's second law: force equals mass times acceleration, one can equate the gravitational force with the quantity $m \mathbf{r}^{\prime \prime}(t)$, so that the mass $m$ of the earth cancels out, and one arrives at equation (1) with the value of the constant $c$ being $c=G M$. Furthermore, if we apply the same reasoning to all the other planets circling the sun, we get the same value of the constant $d$ in (2), as asserted by Kepler's third law.

The flaw in this argument is that in order to use the expression $\mathbf{r}^{\prime \prime}(t)$ for the acceleration, we must be using an inertial frame for our coordinate system. For example, if the sun happened to be accelerating at exactly the same rate as the earth at a given instant, then the acceleration of the displacement vector $\mathbf{r}(t)$ would be zero, even though the force was unchanged. So the implicit assumption being made is that the sun is fixed. One could of course point out that assumption explicitly, together with a remark that it is close enough to being true that the resulting conclusions are very close to being correct. However, one can give a cogent argument that shows clearly why we may assume the sun to be fixed, and also has the advantages mentioned in our introduction-all the hard work is already done in proving the three properties P1-P3 for solutions of equation (1).

The Application of Newton's Laws. Let $\mathbf{X}$ and $\mathbf{Y}$ denote the position vectors of the earth and sun, respectively, in some inertial coordinate system. If $m$ is the mass of the earth, and $M$ the mass of the sun, then the center of gravity $\mathbf{C}$ of the pair is defined by the equation

$$
(m+M) \mathbf{C}=m \mathbf{X}+M \mathbf{Y} .
$$


Let $\mathbf{r}=\mathbf{X}-\mathbf{Y}$ be the displacement vector from the sun to the earth. Then Newton's law of gravity says that the gravitational force $\mathbf{F}$ exerted by the sun on the earth is given by

$$
\mathbf{F}=-G M m \mathbf{r} / r^{3},
$$

while Newton's second law asserts that

$$
\mathbf{F}=m \mathbf{X}^{\prime \prime}(t)
$$

Equating these two expressions gives

$$
\mathbf{X}^{\prime \prime}(t)=-G M \mathbf{r} / r^{3}
$$

In exactly the same way, the gravitational force of the earth on the sun gives

$$
\mathbf{Y}^{\prime \prime}(t)=G m \mathbf{r} / r^{3} .
$$

From equations (4b) and (4c) we see using (4) that $\mathbf{C}^{\prime \prime}(t) \equiv 0$. Hence

$$
\mathbf{C}(t)=t \mathbf{a}+\mathbf{b} \quad \text { for constant vectors } \mathbf{a}, \mathbf{b}
$$

and $\mathbf{C}(t)$ can be used for the origin of a new inertial coordinate system. It also follows by subtracting (4b) from (4c) that

$$
\mathbf{r}^{\prime \prime}(t)=-G(m+M) \mathbf{r} / r^{3} ;
$$

in other words, equation (1) holds for $\mathbf{r}(t)$, with the constant $c=G(m+M)$. We can also write this as $c=M G(1+\mu)$, with $\mu=m / M$. For the earth-sun pair, one has $\mu$ approximately equal to $2 \times 10^{-6}$. Even for Jupiter, $\mu$ is approximately $10^{-3}$ so that using the value $c=M G$ in (1) should not have too great an effect on the solution. In any case, the solutions to equation (5) must satisfy the three properties P1-P3 for any solution of equation (1).

Next, let $\mathbf{x}=\mathbf{X}-\mathbf{C}$ and $\mathbf{y}=\mathbf{Y}-\mathbf{C}$ be the position vectors of the earth and sun referred to their common center of gravity. Then

$$
m \mathbf{x}+M \mathbf{y}=0 \quad \text { and } \quad \mathbf{x}-\mathbf{y}=\mathbf{r}
$$

so that

$$
(m+M) \mathbf{x}=M \mathbf{r} \quad \text { and } \quad(m+M) \mathbf{y}=-m \mathbf{r} .
$$

It follows that the vectors $\mathbf{x}, \mathbf{y}$, and $\mathbf{r}$ are collinear and that the orbit of the earth is just a contraction of the orbit of the displacement vector between the sun and earth. In fact, we can write these equations as

$$
\mathbf{x}=\mathbf{r} /(1+\mu) \quad \text { and } \quad \mathbf{y}=-\mu \mathbf{r} /(1+\mu)
$$

which implies, in particular, that

$$
\mathbf{y}=-\mu \mathbf{x}
$$


Hence the earth and sun both describe an ellipse with one focus at their common center of gravity. Further, both orbits have the same eccentricity and the same period. But since $\mu$ is so small, the orbit of the sun is equally small, and that of the earth very close to that of the displacement vector $\mathbf{r}$. That is the reason that one gets close to the correct answer by assuming that the sun is fixed, and also the reason that neither Galileo nor the Church was exactly right in asserting that the sun or the earth, respectively, was fixed, although Galileo was a lot closer to the truth. However, as minimal as the motion of the sun or of any other star may be in response to the pull of a planet orbiting around it, it is precisely that motion that has led to the conclusion that there are many planets orbiting other stars, even though none of the planets themselves has ever been directly observed. We turn now to a more detailed look at what is observed and what is inferred.

Extrasolar Planets. It is precisely the universality of Newton's universal law of gravity (4a) that tells us that all the equations and conclusions derived for the sun and earth interaction also hold for any system consisting of a star and a single planet orbiting around it, where $M$ and $m$ are the respective masses of the star and planet. In particular, the period $T$ of the planet is the same as the period of the star: the time it takes each of them to complete one orbit around their common center of gravity. But the period is the easiest thing to determine, since precisely what is detected is a periodic motion of the star. (At least that is true in theory; in practice, determining the period from a finite set of observations can prove tricky.) The other quantity that astronomers are usually able to estimate fairly accurately is the mass $M$ of the star, based on the spectral type and luminosity of the star.

The correct version of Kepler's third law, equation (2), uses (3) with the correct value of the constant $c$ in (1): $c=G(M+m)=G M(1+\mu)$, from (5). It follows that the ratio $T^{2} / a^{3}$ is not exactly the same for all planets, but is very close to the value $4 \pi^{2} / G M$, since the ratio $m / M$ is, almost by definition of a planet, very close to zero. As a consequence, the first fact about the unseen planet that we can infer immediately from the knowledge of $M$ and $T$ is its distance from the star-the value $a$ of the semimajor axis of its elliptical orbit:

$$
a^{3}=G M(T / 2 \pi)^{2} .
$$

The determination of the other characteristics and orbit parameters of the planet depends on the method used to observe the motion of the star. A highly readable overview giving the astronomical background and the long search for extrasolar planets, culminating in the first confirmed success in 1991, can be found in [2]; see, in particular, p. 175 and pp. 218-226, for a description and discussion of the relative advantages of the various methods of detection. Almost all the new planets were discovered by detecting variations of the radial velocity of a star, determined by a Doppler shift in its spectrum. These are the ones listed on the exoplanets website.

The Table of Extrasolar Planets. A list and description of all the extrasolar planets discovered since 1995 can be found on the website http://exoplanets.org. This site can also be reached via the University of California at Berkeley astronomy department: http://astron.berkeley.edu/ gmarcy. Figure 1 shows the first few rows of the table. There are six columns:

1. The identity of the central star.

2. A quantity denoted "Msini" described below.

3. The period $T$ of the planet in units of earth days. 
4. The semimajor axis $a$ of the planet's orbit, in astronomical units AU, where $1 \mathrm{AU}$ is the distance from the earth to the sun.

5. The eccentricity of the orbit.

6. A quantity " $K$ " described below, in units of meters per second.

Figure 1. Masses and Orbital Characteristics of Extrasolar Planets. Last Updated 13 August 2000. using stellar masses derived from Hipparcos, metalicity, and stellar evolution. The first 12 extrasolar planets on the list given at the website http://exoplanets.org.

\begin{tabular}{rlccccr}
\hline Star Name & $\begin{array}{c}\text { Msini } \\
\text { (Mjup) }\end{array}$ & $\begin{array}{c}\text { Period } \\
(\mathrm{d})\end{array}$ & $\begin{array}{c}\text { Semimajor } \\
\text { Axis (AU) }\end{array}$ & Eccentricity & $\begin{array}{r}K \\
(\mathrm{~m} / \mathrm{s})\end{array}$ \\
\hline 1 & HD83443 & 0.35 & 2.986 & 0.038 & 0.08 & 56.0 \\
2 & HD46375 & 0.25 & 3.024 & 0.041 & 0.02 & 35.2 \\
3 & HD187123 & 0.54 & 3.097 & 0.042 & 0.01 & 72.0 \\
4 & TauBoo & 4.14 & 3.313 & 0.047 & 0.02 & 474.0 \\
5 & BD-103166 & 0.48 & 3.487 & 0.046 & 0.05 & 60.6 \\
6 & HD75289 & 0.46 & 3.508 & 0.048 & 0.00 & 54.0 \\
7 & HD209458 & 0.63 & 3.524 & 0.046 & 0.02 & 82.0 \\
8 & 51Peg & 0.46 & 4.231 & 0.052 & 0.01 & 55.2 \\
9 & UpsAndb & 0.68 & 4.617 & 0.059 & 0.02 & 70.2 \\
10 & HD168746 & 0.24 & 6.400 & 0.066 & 0.00 & 28.0 \\
11 & HD217107 & 1.29 & 7.130 & 0.072 & 0.14 & 139.7 \\
12 & HD162020 & 13.73 & 8.420 & 0.072 & 0.28 & 1813.0 \\
\hline
\end{tabular}

The method used to obtain the information in columns 2-6 of this table is to measure the Doppler shift to determine the "radial velocity": the rate at which the distance between us and the given star increases or decreases over time. If a planet orbits the star, one should have a periodic change in that rate, except for the extreme case in which the plane of the orbit is perpendicular to our line of sight. In our discussion we assume that the motions of the earth relative to the sun have already been taken into account, as well as any long-term steady change of distance between the star and the sun, which appears as a median line for the periodic variation in radial velocity due to the star's "wobble" caused by the orbiting planet.

In the simplest case of a nearly circular orbit, the planet describes a circle of radius $a$ at constant velocity $v$, and the star describes a circle with constant velocity $V$, both orbits having period $T$. Then $v T=2 \pi a$, and since $T$ determines $a$, by Kepler's third law (7), we also have the velocity $v$ of the planet. Then, from (6) we conclude that $v m=V M$ so that we could determine the mass $m$ of the planet, if we knew the value of $V$. If the plane of the orbit contained our line of sight, then $V$ would simply be the maximal radial velocity. In general, if $i$ denotes the angle of inclination between the normal to the plane of the orbit and our line of sight to the star, then the maximal radial velocity would be the quantity $K=V \sin i$, and hence we can deduce the quantity $m \sin i=K M / v$. These two quantities are given in the last and second columns of the table, respectively. In particular, the second column, "Msini", represents the mass of the planet times the sine of the angle of inclination of its orbit, and hence provides the minimum mass for the planet. The unit used is the mass of Jupiter.

When the orbit is seriously elliptical, rather than nearly circular, then everything is much more complicated. In addition to the inclination of the plane of the orbit, 

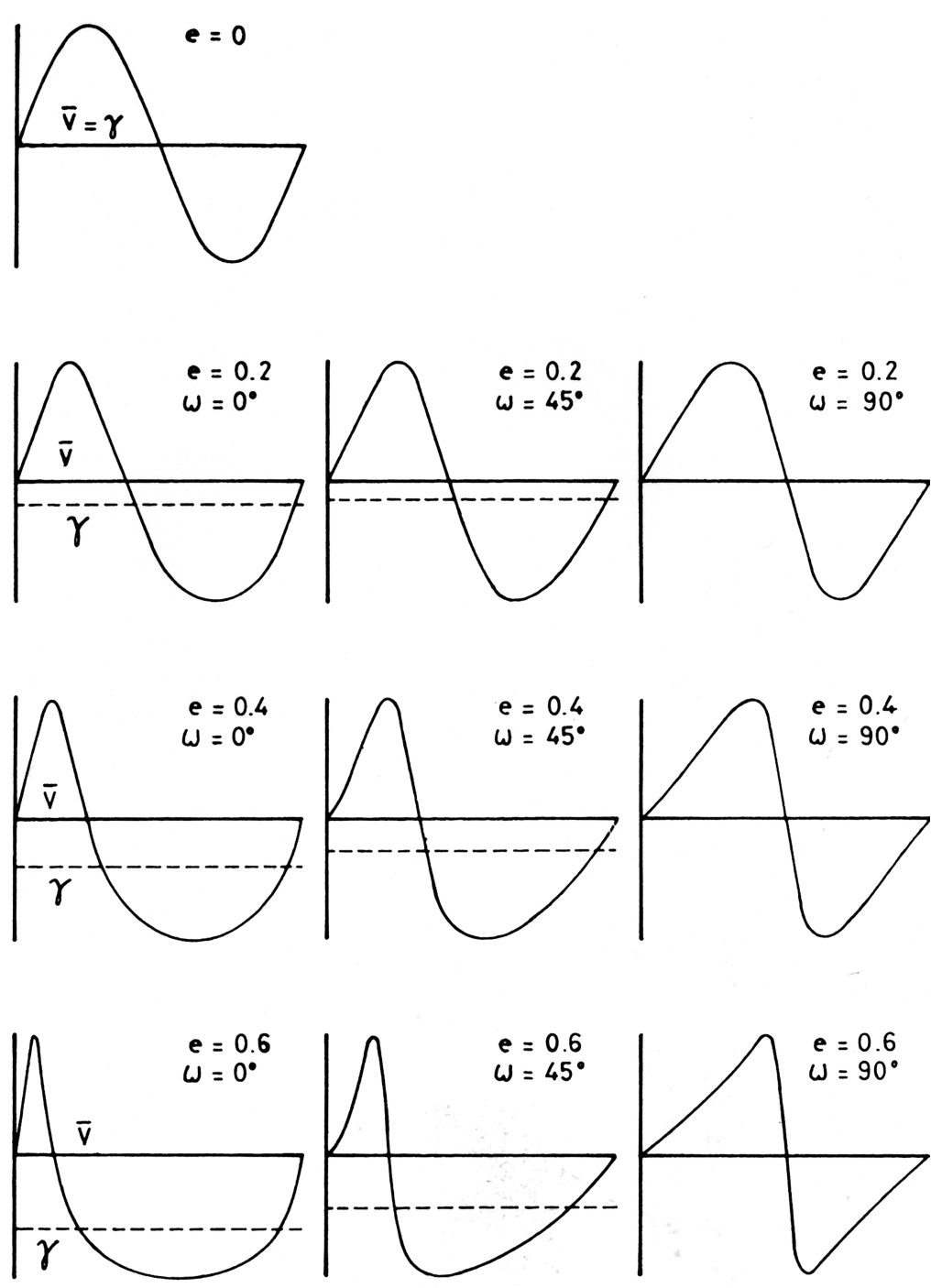

Figure 2. A sample of radial velocity curves showing the variation in shape resulting from different values of the eccentricity $e$ of the orbit and the orientation of the major axis of the orbit with respect to our line of sight. From [3, p. 83]. Reproduced by courtesy of the author.

there are two additional parameters: the orientation of the ellipse in that plane and the value of the eccentricity. It turns out that as these parameters vary, the shape of the curve that describes the variation in radial velocity with respect to time gets distorted in certain distinctive ways from the sine curve of circular motion. Figure 2 gives a sample of these. One can do a best-fit approximation to determine the unknown parameters (except for the angle of inclination, whose sine enters into the formulas, but is itself not obtainable). More information about these matters can be found on links given at the exoplanet website.

Concluding Remarks. It may seem a bit finicky to resist accepting the sun as fixed when the conclusion of the argument shows that to be essentially correct. However, in addition to the reasons already spelled out, there are several further considerations. 
First, the two-body problem applies not only to planets around stars, but also to the motion of binary stars, which are very abundant and also very important in astronomy. In that case, it would be totally incorrect to assume that either one is fixed, but the correct treatment of the earth-sun motion applies without change; see [3].

Second, this subject provides a good opportunity to say a few words about what is involved physically in the choice of coordinate systems. One is quite free to choose them conveniently for mathematical purposes, but if we are treating a problem in physics, then we must be aware of the physical consequences. In particular, inertial coordinates play a preferred role both in Newtonian physics and in special relativity.

Third, although considerations of the general theory of relativity go far beyond the limits of this discussion, there are a few remarks worth making. In particular, although we have referred somewhat facetiously to the case of Galileo and the Church, it is a fact that few people really understand the issues involved, and there is a tendency to dismiss the whole debate as irrelevant, both for physical reasons - that in fact we now know that everything is moving: the entire solar system is circling the galaxy, the whole galaxy is moving with respect to other galaxies, and roughly speaking, "all motion is relative" - and also for the mathematical reason that we can choose coordinates in which anything we like is fixed. However, on the practical side, there is at least one issue on which the Church agreed with the Copernicans: the question of whether the Earth revolved around the Sun or vice versa should be decidable relative to the background of the sphere of fixed stars. That did indeed happen, but not until hundreds of years later when it became possible to measure the parallax due to the Earth's motion. And on the theoretical side, general relativity does indeed play an ever-increasing role in celestial mechanics, but it does not change our discussion in any essential way. The reader who wishes to pursue this aspect further can consult [1].

Finally, there is a somewhat misleading way in which many books present the Kepler and Newton Laws, implying that they are essentially equivalent: that Kepler's laws imply Newton's Laws and vice versa. But neither implication is accurate. It is true that Newton used Kepler's Laws in his reasoning, but his starting point was that there should be dynamical laws involving forces that determine the orbits of planets (among other things) and what Kepler's laws imply is that if one has such laws, then they must be of a certain form. In no way do Kepler's Laws imply the existence of Newton-type laws. And conversely, as we have seen, Newton's Laws imply something that is both more and different from Kepler's original laws.

Also a final word about units. As with coordinates, one has a lot of freedom in their choice, but one must be careful to keep track. In the case of the simplified form of Kepler's third law (7), the right-hand side of the equation

$$
a^{3} / T^{2}=G M / 4 \pi^{2}
$$

must equal 1 when the units used are earth-years for the period $T$ and astronomical units AU for the quantity $a$, with $M$ equal the mass of the sun. But then using the same units for a planet around any other star, the right-hand side of (8) reduces simply to $M^{*}$ : the mass of the star expressed in units of solar masses.

ACKNOWLEDGEMENTS. I thank leading planet hunter Geoff Marcy for establishing and overseeing the exoplanet website, and for his helpful comments while I was preparing this article. Also Ken Croswell, whose book Planet Quest was extremely useful in providing the astronomical background and who corrected some misstatements in my first draft. Barrett O'Neill, David Gilbarg, Hans Samelson, and Sherman Stein contributed clarifications for which I am grateful. 


\section{REFERENCES}

1. V. A. Brumberg, Essential Relativistic Celestial Mechanics, Adam Hilger, Philadedelphia, 1991.

2. K. Croswell, Planet Quest, Harcourt Brace, New York, 1997.

3. W. D. Heintz, Double Stars, D. Reidel, Dordrecht, 1978.

ROBERT OSSERMAN received his Ph.D. from Harvard University under the direction of Lars V. Ahlfors. He then joined the faculty of Stanford University, where he is now Professor Emeritus. Since 1990, he has been associated in different capacities with MSRI, currently as Special Projects Director. His research interests have ranged from complex function theory and Riemann surfaces to minimal surfaces and other aspects of differential geometry. He has had an amateur interest in astronomy ever since watching the stars from a troopship crossing the Pacific in 1946. The two interests combined led to his writing the book, Poetry of the Universe: $a$ Mathematical Exploration of the Cosmos, as well as the present paper.

Mathematical Sciences Research Institute, 1000 Centennial Drive, Berkeley, CA 94720-5070

ro@msri.org 